\title{
Neutron Radii in Mean-Field Models
}

\author{
R. J. Furnstahl用 \\ Department of Physics \\ The Ohio State University, \\ Columbus, OH 43210
}

(Dated: December, 2001)

\begin{abstract}
Bulk nuclear observables such as charge radii and binding energies are well described by both nonrelativistic and covariant mean-field models. However, predictions of neutron radii, which are not tightly constrained by reliable data, vary significantly. The nature of this variation is investigated using correlations between basic properties of the models and the neutron skin thickness in lead. The results suggest that conventional covariant models are too limited. The study is guided by principles and insights of effective field theory (EFT), such as power counting, and the relation of mean-field models to a more general EFT approach to nuclei is discussed.
\end{abstract}

PACS numbers: 21.10.Gv,21.30.Fe,21.60.-n

*Electronic address: furnstahl.1@osu.edu 


\section{INTRODUCTION}

Bulk nuclear observables such as charge radii and binding energies are well described by both nonrelativistic and covariant mean-field models [1, 2]. Yet the "best fit" calculations of each type in the literature disagree substantially on neutron radii [1, 2, 3]. The spread in mean-field predictions and a recent analysis of experimental determinations [3] suggest that neutron radii in medium to heavy nuclei are inadequately determined at present by either theory or experiment. Better values for neutron radii could have widespread impact, from reducing uncertainties in atomic parity violation experiments [3] to constraining properties of the surface crust of neutron stars [4]. More generally, the lack of control over neutron radius predictions implies large uncertainties in extrapolations to neutron- or proton-rich nuclei, which play an important role in nuclear astrophysics [5].

There are arguments in the literature that mean-field models of nuclei are inadequate because the mean-field approximation is not valid at ordinary nuclear densities or that essential correlations are excluded [6]. However, these are misleading arguments, which implicitly assume that one is performing a Hartree or Hartree-Fock calculation of some underlying interaction. In fact, it is more appropriate to identify these calculations with Kohn-Sham density functional theory (DFT) [7, 8, 9], in which the full Hartree plus exchange-correlation functionals (and not Hartree alone!) are approximated by a parametrized form.* The important observation is that mean-field models are specified by a universal energy functional of the density, which is minimized iteratively for each nucleus through the introduction of auxiliary single-particle orbitals. This is the structure of a Kohn-Sham DFT. The solution procedure takes a small fraction of the computational effort of conventional direct manybody solutions. The universality is important because the difficulties in finding solutions to many different nuclei are shifted to constructing the functional once; if the functional is easily evaluated then the cost of subsequent applications to each additional finite nucleus is small, and one can scale to large numbers of nucleons.

"Mean field" in this context really implies a limited form of the analytic and nonlocal structure that can appear in the exact energy functional. We interpret the mean-field models as a form of Kohn-Sham DFT in which the functional is approximated by a type of generalized gradient expansion [13, 14]. From this point of view, the underlying framework is completely general and the limitations of present-day mean-field calculations could be systematically removed without losing the computational advantages of the approach. Deficiencies in the mean-field model predictions should therefore be traced to deficiencies in the approximate functional rather than to a breakdown of the underlying framework.

In this paper, we adapt this philosophy to a study of mean-field neutron radii. The nature of the variation in radii is investigated using correlations between basic characteristics of the models and the predicted neutron skin thickness in lead. In this way, we can identify how properties of the mean-field energy functionals are related to observables and identify possible deficiencies in the functional. The study is guided by the principles and insights of effective field theory (EFT), with an eye on the ultimate goal of embedding mean-field models into a more general DFT/EFT approach to nuclei [15. With an EFT approach one can exploit power counting to systematically approximate the energy functional. In the present context, power counting means that one can estimate the contribution to the energy

\footnotetext{
* The observation that mean-field models can be interpreted as Kohn-Sham functionals has been made by Brack for Skyrme models 10] and by Schmid et al. for covariant models 11, 12.
} 
of individual terms in the energy functional, even those excluded by the model truncation. The promise of this approach is supported by empirical evidence that EFT power counting in mean-field models is robust [16, 17, 18, 19].

In principle, a detailed analysis during the fitting process would best reveal how observables such as the neutron radius are correlated with other properties of the model. As an alternative, we consider a large set of models all fit to roughly the same data at about the same accuracy, and then examine the correlations graphically. This strategy has been used with covariant models to study deformations in nuclei [20] and spin-orbit splittings [21]. Brown has used such correlations with the Skyrme model recently to address many of the same issues about neutron radii [22] and Typel and Brown have extended these results to conventional covariant models [23]. In our analysis, we use conventional parameter sets from the literature but also generalized functionals developed for EFT studies. The latter provide a wide class of models that provide very good fits to the usual bulk nuclear data but which also span a wide range of observables that are not well constrained by this data.

We find that the neutron skin thickness is dominantly correlated with the bulk symmetry energy and its density dependence. Thus the properties of asymmetric infinite nuclear matter (as opposed to surface properties) are most important. Our conclusions on the physics determining the skin are in accord with many of the observations in Ref. [24], which are based on applying a macroscopic model and comparing to two particular mean-field models, and by Brown [22] and Brown and Typel [23]. (Note: many of the same issues were discussed long ago by Bodmer who used a semi-empirical statistical method [25]. Other recent studies of the neutron skin in mean-field models include Refs. [26, 27, 28, 29, 30].) The differences between predictions from standard Skyrme and covariant models can then be traced to differences in the density dependence of the symmetry energy. A comparison of the symmetry energy derived from more microscopic approaches that are tied to free-space nucleon-nucleon scattering implies that conventional covariant models based on heavy meson interactions are too simple.

The plan of the paper is as follows. In Sect. II), we describe the mean-field functionals and and in Sect. III the EFT power counting. The correlation analysis is carried out in Sect. IV and the consequences discussed in Sect. V. Sect. VI is a summary.

\section{OVERVIEW OF MEAN-FIELD ENERGY FUNCTIONALS}

There are two major classes of mean-field models in general use, which can be formulated in terms of nonrelativistic (Skyrme) or covariant energy functionals. The covariant models can be further subdivided into meson and point-coupling models. We will compare all three types in our correlation analysis. These models and their implementations are well documented in the literature [1, 2, 17, 19, 31, 32, 33, 34, 35, 36, 37, 38, 39, 40] and we will only discuss here details relevant to the present investigation. We focus directly on energy functionals instead of Hamiltonians or Lagrangians to stress the connection to a more general formulation in terms of density functional theory. The usual construction of the functional starts by postulating interactions (either a Skyrme effective interaction or a Lagrangian) and then treats them in the Hartree approximation. ${ }^{\dagger}$

\footnotetext{
$\dagger$ Note that the Skyrme models actually apply a Hartree-Fock approximation, but since the forces are zero range, this doesn't affect the form of terms in the energy functional.
} 
The Skyrme models are based on nonrelativistic zero-range, density-dependent interactions. The energy functional most commonly used in the present-day literature has evolved only slightly from the original form proposed; an explicit expression for the interaction is given in Ref. [G]. The corresponding functional can be written as powers and gradients of the isoscalar density $\rho=\rho_{p}+\rho_{n}$ and isovector density $\rho_{3}=\rho_{p}-\rho_{n}$, the corresponding kinetic energy densities $\tau$ and $\tau_{3}$, and spin-orbit currents. As such it can be considered a Kohn-Sham functional constructed as a generalized gradient expansion [13, [14]. Of particular note are terms proportional to $\rho^{2+\alpha}$ and $\rho^{\alpha} \rho_{3}^{2}$, which in modern Skyrme parameter sets appear with fractional $\alpha$. Although extended Skyrme models have been considered [31], the density expansion stops with these terms. There are usually four isovector parameters (which are linear combinations of the traditional parameters $t_{0}-t_{3}, x_{0}-x_{4}$, and $W_{0}$ [1]), corresponding to $\rho_{3}^{2}, \rho^{\alpha} \rho_{3}^{2}, \rho_{3} \tau_{3}$, and $\left(\nabla \rho_{3}\right)^{2}$ terms (there are also isovector terms involving the spin-orbit currents). While the Skyrme interaction has been motivated in terms of underlying nucleon-nucleon interactions 41, 42], the connection has only been made qualitatively and the parameters are treated phenomenologically. A goal of the EFT/DFT program advocated here will be to revisit this connection and seek systematic improvement.

Many Skyrme parameter sets are available in the literature [22, 32, 33, 34, representing a wide range of fits to bulk nuclear properties, although the fits are not all to the same set of observables and are not of equal quality. The Skyrme parameter set developed by Brown [1] is fit to the widest range of properties and serves as the quality-of-fit benchmark for this class of models. We will use most of the sets from the literature plus many new ones generated for this investigation. The new sets are fit to binding energies, charge densities, and spin-orbit splittings of doubly magic nuclei as described for covariant models in Refs. [19] and [17]. However, a value for the neutron radius in lead was also specified so as to generate sets that cover as large a range in neutron radii as is compatible with a good fit to the other properties. Only new sets with a quality of fit comparable to Ref. [1] were used in our analysis.

The most commonly used covariant mean-field models are derived from Lagrangians with nucleons coupled to heavy mesons and are characterized by large, isoscalar, mean meson fields that are several hundred $\mathrm{MeV}$ in magnitude [2, 35, 36, 37]. Meson exchange in these models is spacelike and far off-shell, so the corresponding parameters are at best only roughly related to the physical meson spectrum. We describe these energy functionals as "covariant" rather than "relativistic," as is conventional in the literature, since the latter suggests the importance of kinematic relativity - large velocities and large $p / M$-whereas these corrections are small for nuclei. Rather it is the potentials where relativity is most important, and this is manifested in a Lorentz covariant representation, which maintains the distinction between Lorentz scalars and zeroth components of four-vectors 443]. (It is not Lorentz invariant, since the four velocity of a nucleus or nuclear matter defines a preferred frame.)

In practice, the form of the energy functional has been limited to terms that can arise from the one-loop or Hartree approximation to a Lorentz invariant Lagrangian. In this sense the functionals are less general than the Skyrme functionals. This comparison is somewhat misleading, however, since a functional built from integral powers of both Lorentz scalar and vector densities will, when reduced to nonrelativistic form, contain terms non-analytic in the density (i.e., analogous to the $\rho^{2+\alpha}$ term for particular values of $\alpha$ ) [37, 44]. There are several standard covariant-model parameter sets in the literature [38, 39] and many more have been generated recently in the course of EFT investigations [19, 45]. We use them 
all in the present investigation. In conventional mean-field parametrizations, the model is usually truncated to minimize the parameters needed to provide a good fit, with the hope of maximizing predictive power. Terms through the fourth power of the scalar mean field are included but only through the second power of the other mean fields; there is only one non-gradient isovector parameter. In an EFT-inspired analysis, one uses power counting arguments to organize the energy functional and reveal how many parameters can be fixed from the data. The EFT parameter sets include more interaction terms but are still not complete. For example, a meson corresponding to isovector scalar exchange was not considered because its effects were found to be small in previous investigations. In Ref. [46], this is argued to have an important effect on the symmetry energy.

An alternative to explicit mesons are "point coupling" (PC) models, which feature contact interactions and derivatives among nucleons only rather than meson exchange [17, 40, 47]. The energy functional here is composed of powers and gradients of Lorenz scalar $\left(\rho_{s}\right)$, vector $\left(\rho_{B}\right)$ and tensor densities and currents [16]. Point coupling models are somewhat more flexible than covariant meson models because the assumption of an underlying meson exchange does not constrain terms in the energy functional. Thus a term such as $\left(\nabla \rho_{s}\right)^{2}$ would have a definite sign if based on an underlying meson exchange but could have either sign in a point-coupling model.

In both types of covariant models, the isovector interactions in conventional formulations are quite limited, with the form of simple rho meson exchange or its point-coupling equivalent. Since the corresponding mass is largely unconstrained by fits to nuclear properties, there is really only one free parameter. This is in contrast to conventional Skyrme interactions, which have up to four parameters controlling isovector contributions (although two are largely irrelevant for the properties discussed here [1]).

\section{POWER COUNTING IN THE ENERGY FUNCTIONAL}

An effective field theory (EFT) describes low-energy physics with low-energy degrees of freedom. Underlying EFT is the principle that while the short-distance, ultraviolet behavior of the effective theory may be incorrect, it can be corrected systematically by the renormalization of local operators ("counterterms") 48]. There is considerable freedom in the choice of representation of these operators; while one choice is not more "correct" than another, it may be more convergent or easier to calculate. It is in this context that we compare nonrelativistic and covariant energy functionals of the density for nuclei. A summary of the EFT philosophy as applied to mean-field models is given in Ref. [15]. For our purposes, the most important concept is that of power counting in the energy functional, which associates a natural size to every term based on conjectured underlying scales.

Will an EFT approach be useful for heavy nuclei? An EFT relies on the separation of scales, so we should identify the momentum scale $\Lambda$ that divides short from long-distance physics. Long-distance physics must be treated explicitly while short-distance physics can be reproduced with local operators. For the latter we might expect a density expansion in powers of $k_{\mathrm{F}} / \Lambda$, where $k_{\mathrm{F}}$ is the Fermi momentum. (This is manifest in the treatment of a dilute Fermi system [49], but has not been shown for nuclear systems.) There are two immediate problems. First, nuclear matter equilibrium $k_{\mathrm{F}}$ is significantly larger than the pion mass, which implies that an explicit treatment of pion physics as long-range physics is essential for a useful EFT. Second, nuclear matter saturation might only occur when $k_{\mathrm{F}} \approx \Lambda$, because only then could different terms in the density expansion compensate each other. 
However, the bulk physics of heavy nuclei is dominated by isoscalar physics (see below), and the dominant pion contributions appear to be two-pion physics, which is associated with the Lorentz scalar " $\sigma$ " meson [36]. The success in using $\sigma$ exchange in two-nucleon potentials implies that the low-energy tail of the two-pion contribution is not well resolved, particularly in nuclei. ${ }^{\ddagger}$ The consequence is that in an EFT, nuclear structure is dominated by short-distance contact terms ("low-energy constants"), which implies that a model with $\Lambda \approx 600 \mathrm{MeV}$ without explicit pions is actually a good starting point. This argument also implies that isovector observables such as the neutron skin, which could be sensitive to one-pion-exchange physics, may be inadequately described.

The potential problem with applying EFT to saturating systems, in which saturation is only possible at the breakdown scale, is apparently not realized in nuclei. In fact, by conventional analysis, equilibrium nuclear matter is an anomalously dilute Fermi system. One such analysis, by Jackson [51, 52], is based on saturation driven by a strongly repulsive short-range interaction (which we'll call a "hard core") with range $c$ (so that $\Lambda \approx 1 / c$ ). The size $R$ of a saturating system scales with the number of particles $A$ to the one-third power, which defines a characteristic length scale $r_{0}$ :

$$
R \sim r_{0} A^{1 / 3}
$$

Jackson estimates the limits of saturation to be bounded by $0.552 c \leq r_{0} \leq 2.4 c$ [51]. This estimate is consistent with liquid ${ }^{3} \mathrm{He}$, where $r_{0} \approx 0.96 c$, but with a nuclear hard core at $c=0.4 \mathrm{fm}, r_{0}$ is actually beyond the bound: $r_{0} \approx 2.75 c$, which implies that saturation in nuclei is unconventional. One possible explanation for saturation in an EFT with a density expansion is that two low orders might largely cancel because of an anomalously small coefficient multiplying a leading term, while all higher orders follow the expansion in $k_{\mathrm{F}} / \Lambda$. This is the mechanism for saturation in covariant mean-field models [15].

We assume that a counting consistent with low-energy QCD, including pions as light degrees of freedom, is appropriate for our mean-field energy functionals. Georgi-Manohar naive dimensional analysis, or NDA, assigns appropriate powers of the pion decay constant $f_{\pi} \approx 93 \mathrm{MeV}$ and a scale of non-goldstone boson physics $\Lambda$ (about $600 \mathrm{MeV}$ ) to terms in a low-energy effective Lagrangian of QCD [53, 54]. The counting for a generic term in a covariant meson Lagrangian is found from

$$
\beta\left[\Lambda^{4} / g^{2}\right]\left(\frac{g^{2} \bar{\psi} \psi}{\Lambda^{3}}\right)^{l}\left(\frac{\nabla}{\Lambda}\right)^{p} \frac{1}{m !}\left(\frac{g \phi}{\Lambda}\right)^{m} \frac{1}{n !}\left(\frac{g V}{\Lambda}\right)^{n}
$$

where $g \sim \Lambda / f_{\pi}$ and Dirac and isospin operators are not shown. (See Ref. 19] for a discussion of power counting with covariant meson Lagrangians including pions.) The assumption here is that this power counting, intended for terms in a Lagrangian, can be applied directly to the energy functional through the associations $\rho_{\mathrm{s}} \leftrightarrow \bar{\psi} \psi, \rho_{\mathrm{B}} \leftrightarrow \psi^{\dagger} \psi$ and so on. This connection is immediate if the mean-field model is viewed as being only a one-loop calculation but has not been fully clarified in the more general context of DFT. Skyrme functionals follow an analogous power counting behavior with powers of $\rho$ and $\tau$ counted using $\rho \leftrightarrow \psi^{\dagger} \psi$, $\tau \leftrightarrow(\nabla \psi)^{2}$, and so on (see Ref. [18] for details).

If we have identified the appropriate underlying length scales, naturalness implies that the dimensionless constant $\beta$ is of order unity. Given this result, we can estimate contributions

\footnotetext{
$\ddagger$ Indeed, one must work very carefully to discern the long-range chiral two-pion-exchange tail in the detailed two-nucleon scattering data [50].
} 


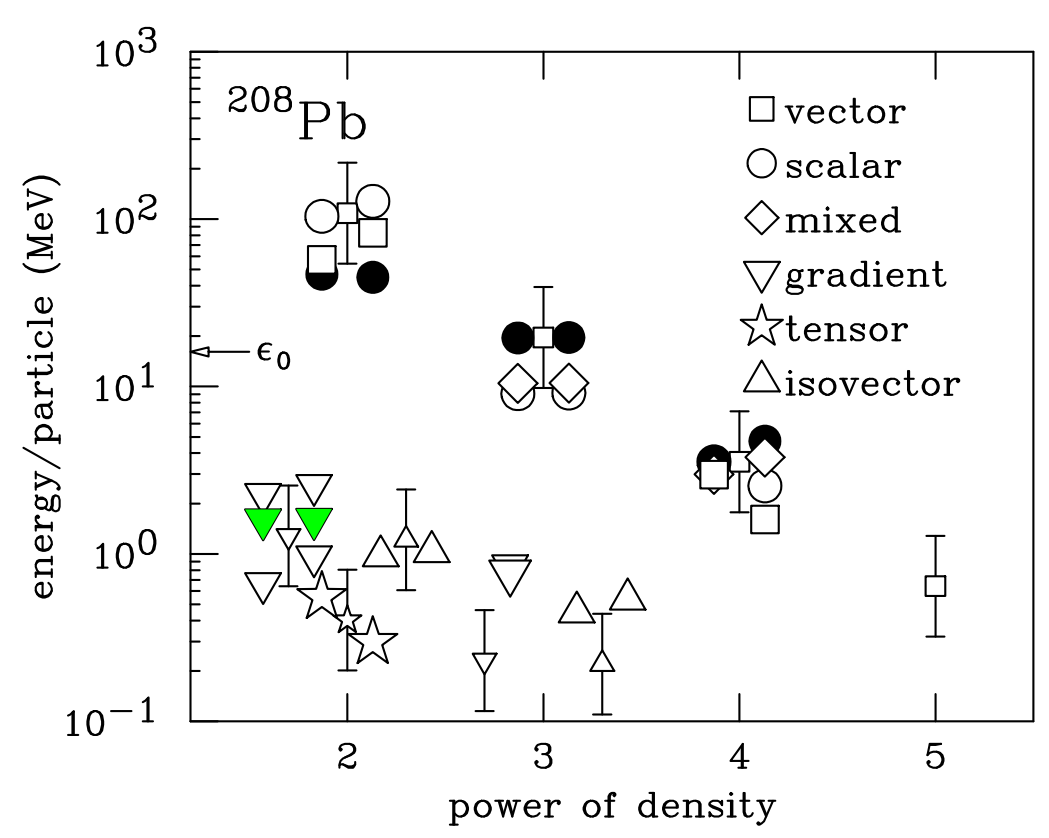

FIG. 1: Contributions to the energy per particle in ${ }^{208} \mathrm{~Pb}$ for two covariant point-coupling models from Ref. [17]. Absolute values are shown and the filled symbols are net values for the sum of scalar and vector terms. The small symbols indicate estimates based on NDA [Eq. (3)], with the error bars corresponding to natural coefficients from $1 / 2$ to 2 .

to the energy from any term in the mean-field energy functional. For example, a localdensity estimate (per nucleon) for a general isoscalar term with dimensionless coefficient $\beta$ can be made according to

$$
\frac{1}{A} f_{\pi}^{2} \Lambda^{2} \int d^{3} x \beta\left(\widetilde{\rho}_{\mathrm{s}}\right)^{i}\left(\widetilde{\rho}_{\mathrm{B}}\right)^{j} \approx \beta \Lambda\left(\frac{\left\langle\rho_{\mathrm{B}}\right\rangle}{f_{\pi}^{2} \Lambda}\right)^{i+j-1}
$$

where $\widetilde{\rho}_{\mathrm{s}}$ and $\widetilde{\rho}_{\mathrm{B}}$ are naturally scaled scalar and baryon densities (e.g., $\left.\widetilde{\rho}_{\mathrm{s}} \equiv \rho_{s} / f_{\pi}^{2} \Lambda\right)$ and $\left\langle\rho_{\mathrm{B}}\right\rangle$ is the average density in the nucleus being considered. We take $1 / 2<\beta<2$ as a reasonable range of natural coefficients. Other local density prescriptions are detailed in Refs. [16] and [21]. Figure 1 shows these natural estimates with the associated error bars from the variation in $\beta$ as small squares and triangles for ${ }^{208} \mathrm{~Pb}$. Actual contributions to the energy per particle in lead for two covariant point-coupling models that fit nuclear properties are obtained by applying the Hellmann-Feynman theorem [16]. The NDA estimates are validated by these results and many other studies [17, 18, 19, 40, 47, 55, 56].

We will take as a guiding principle that NDA and naturalness are valid for mean-field energy functionals. Indeed, all of the best-fit models exhibit naturalness in all or almost all coefficients. A comparison of goodness-of-fit with this counting at different levels of truncation reveals the limit of the energy in constraining terms. In particular, contributions of order $1 \mathrm{MeV}$ are barely constrained, so contributions from beyond the fourth power of isoscalar densities or meson mean fields are not distinguished from numerical noise [16]. These results explain why the conventional covariant mean-field truncation is phenomenologically successful. In Ref. [16], a complete analysis like this concluded that only six constants in covariant energy functionals are well determined by fits to the usual bulk properties of nuclei. These 
includes four isoscalar terms, one gradient term, and one isovector term. Brown concludes that a similar number of parameters are determined in fits to Skyrme models that use only the standard observables [1].

The conventional set of observables used to fit parameters directly constrains the isovector contribution only through the binding energy (i.e., the symmetry energy). Isovector contributions can be reliably estimated using $\left\langle\rho_{3}\right\rangle \approx(Z-N) / A \times\left\langle\rho_{\mathrm{B}}\right\rangle$. The leading isovector contribution in Fig. 1 is therefore suppressed relative to the leading isoscalar contribution by the factor $[(Z-N) / 2 A]^{2}$ (the extra $1 / 2$ comes from $\frac{1}{2} \tau_{3}$ [16]), which is small even in lead. This puts the leading term on the order of $1-2 \mathrm{MeV}$, which is just resolved by the fits $\left(\rho_{\mathrm{B}}^{5}\right.$ isoscalar contributions are not resolved). When more than one parameter is available, as shown in the figure, only a linear combination is determined, and the subleading coefficient is borderline unnatural [17]. While individual values for these couplings vary widely between different parameter sets, the linear combination is well determined. The power counting analysis supports previous observations in meson models that, at the mean-field level, the role of the $\rho$ meson is simply to adjust the symmetry energy [57, 58].

We emphasize that despite the phenomenological success of the power counting, we do not as yet have a systematic EFT expansion for the energy functionals. In particular, long-range loop contributions may lead to essential nonlocalities and nonanalyticities in the energy functional. An underlying assumption that we are testing is that long-distance contributions do not disturb the power counting. One should not assume, however, that long distance degrees of freedom imply that the functional cannot take the form of powers of densities and gradients. For example, we note that Coulomb systems, which depend entirely on long range physics, are well described by energy functionals based on local density and gradient expansions [9].

\section{CORRELATION ANALYSIS}

Most observables in finite nuclei have a highly nonlinear and correlated dependence on the parameters of the energy functional. Predicting the correlations between features of the mean-field functional and observables such as the neutron radius or the neutron skin thickness is prone to error and plausible explanations often turn out to be incorrect (as illustrated below for finite range effects). This is where power counting and EFT arguments can be illuminating, even in the absence of a systematic EFT expansion. One can also go wrong by considering only a few "best fit" parameter sets, which can lead to false conclusions because of the limited sampling.

Our strategy is to use many parameter sets, each a good fit to bulk properties of doubly magic nuclei. We consider generalized versions of each type of mean-field model and look at correlations between observables and characteristics of the models. In order to fill in gaps that can obscure trends, we also force particular neutron radii, being careful to keep only sets with acceptably good fits to the standard properties. We leave open the question of whether other finite nucleus observables (such as inelastic scattering to collective states) can further restrict the acceptable sets.

In the correlation plots that follow, the shape of a symbol indicates the type of meanfield model: circles for Skyrme, squares for covariant meson, and triangles for covariant point coupling. The shading indicates the origin of the model. Black filled symbols are standard parameter sets from the literature [22, 32, 33, 34, 38, 39, 40. Grey filled symbols are parameter sets generated for EFT investigations [17, 19] and diagonal striped symbols 
are unpublished generalized EFT sets from Ref. [45]. Black and white checkered symbols are new parameter sets generated for this investigation. Other than this (partial) identification we treat all sets democratically. Note that not all models are plotted on all figures.

Comparisons between neutron radii or neutron skins from any given Skyrme model and any given covariant model over a wide range of nucleon number $A$ show that the differences are very systematic. Fluctuations in the difference for particular nuclei, which are affected by details such as the treatment of pairing in open-shell nuclei, are small compared to the difference itself [3, 32]. We conclude that understanding the skin thickness for one convenient nucleus is sufficient at the resolution of this investigation. We focus on ${ }^{208} \mathrm{~Pb}$ since it has the largest skin of the doubly magic nuclei.

The skin thickness is a more robust isovector observable than the neutron radius alone. In principle, the charge radius of $\mathrm{Pb}^{208}$ is very well determined as are the factors that "remove" the charge form factor of the proton (and other smaller corrections). Thus, the point proton radius $r_{p}$ should be very well fixed, and therefore the skin thickness should be closely correlated to $r_{n}$. In practice, when $r_{n}-r_{p}$ is plotted as a function of $r_{n}$ for conventional models, there is a considerable spread, as seen in Fig. 2. This spread reflects slightly different form factor corrections used in the models and differences in how well $\left\langle r^{2}\right\rangle_{\text {ch }}$ in ${ }^{208} \mathrm{~Pb}$ is reproduced by the fits. We want to isolate isovector properties, so the skin thickness is more informative than the individual radii. Since the neutron radii are not included in the fits determining these sets, the scatter is primarily driven by the binding energies (which reflect the bulk symmetry energy).

Thus, we focus on the neutron skin thickness, $\left(r_{n}-r_{p}\right)$, in ${ }^{208} \mathrm{~Pb}$ as a representative isovector "observable" for our study. We put observable in quotes here because, in fact, neither the point proton radius, the point neutron radius, nor their difference is actually an experimental observable. Although it is standard practice, the "unfolding" of a point proton radius from a charge radius measurement is necessarily model dependent. [See Ref. [59] for a discussion from the EFT point of view of the ambiguities in other quantities (occupation numbers) that are often treated as observables.] We will assume that the natural size of this ambiguity is small compared to the size of the difference in neutron radii, but this should be investigated further. A consistent EFT framework will obviate the need to discuss point data, as illustrated in Ref. [19], where nucleon charge form factors are built into the Lagrangian and energy functional.

We also note that the neutron radius is very highly correlated with the neutron form factor at low momentum transfer. This correlation is important for a proposed parity violation experiment at Jefferson Lab, which seeks to make a five percent measurement of the form factor at a momentum transfer of $0.45 \mathrm{fm}^{-1}$. An analysis of the experiment is given by Horowitz et al. [3]. The relationship between the form factor at this momentum transfer and

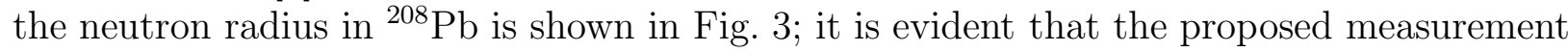
will directly constrain the neutron radius.

These figures already show that conventional covariant meson models (black squares) in general predict larger skin thicknesses than conventional Skyrme models (black circles). A plausible explanation for the larger neutron skin thickness in meson models compared to Skyrme is the finite range of the interaction in the former. The argument is that finite range meson exchange enables the neutron density to extend further than the proton density and still feel the attraction from the protons. However, this picture is not consistent with an EFT viewpoint. The resolution scale associated with meson exchange in the covariant models is $\Lambda \approx 600 \mathrm{MeV}$. For momenta small compared to this scale, the details of the meson exchange 


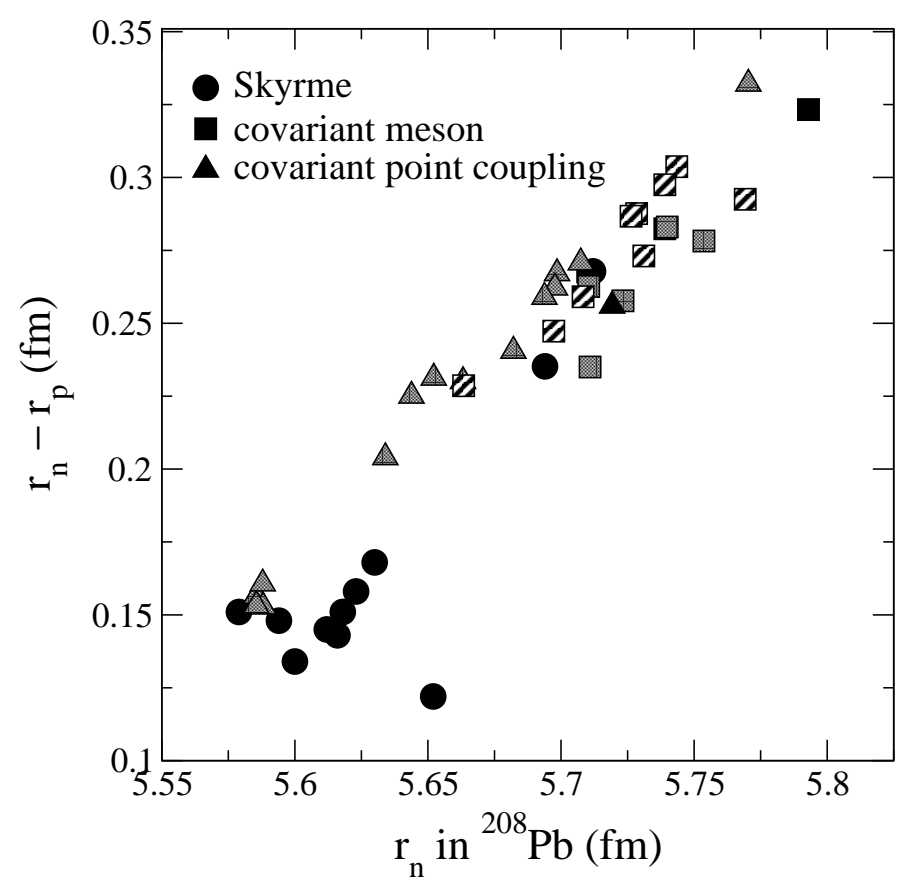

FIG. 2: Neutron skin thickness vs. neutron radius for a wide variety of mean-field models, as described in the text. The shading indicates the origin of the model. Black filled symbols are standard parameter sets from the literature [22, 32, 33, 34, 38, 39, 40]. Grey filled symbols are parameter sets generated for EFT investigations [17, 19] and diagonal striped symbols are unpublished generalized EFT sets from Ref. [45].

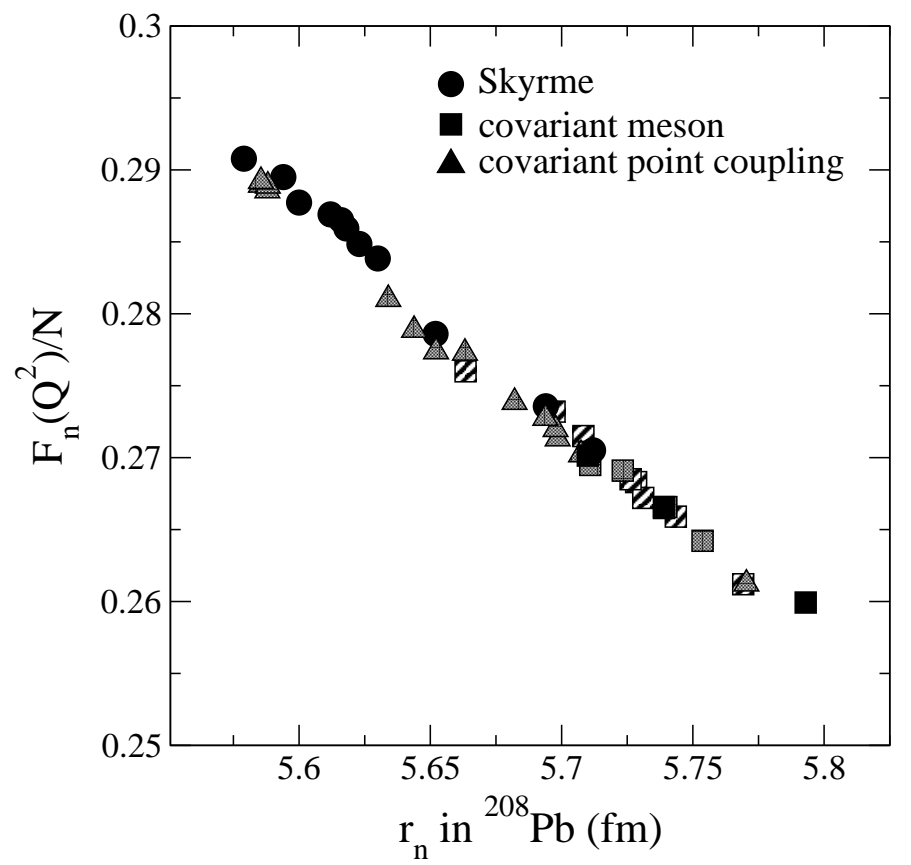

FIG. 3: The calculated neutron form factor (as defined in Ref. [3]) for ${ }^{208} \mathrm{~Pb}$ at momentum transfer $Q=0.45 \mathrm{fm}^{-1}$ vs. the point neutron radius in ${ }^{208} \mathrm{~Pb}$ for a wide variety of Skyrme and covariant mean-field models, as described in the text and in the caption to Fig. 2. 
and the substructure of the hadrons are not resolved. As a result, this short-range physics can be incorporated into the coefficients of operators organized as a derivative expansion. The consequence for low-momentum properties such as the rms radii is that one cannot distinguish interactions with explicit ranges of order $\Lambda^{-1}$ from interactions composed of $\delta$ functions plus derivatives. In principle, one needs an infinite number of terms for complete equivalence; in practice power counting tells us we can truncate sharply.

At tree level, which corresponds to treating the functional as arising from the Hartree approximation, the analysis is trivial. The static propagator of a meson with mass $m \sim \Lambda$ can be expanded in a Taylor series about $|\mathbf{q}|^{2} \equiv q^{2}=0$ :

$$
\frac{m^{2}}{q^{2}+m^{2}}=1-\frac{q^{2}}{m^{2}}+\mathcal{O}\left(q^{4} / m^{4}\right) \approx 1-\frac{q^{2}}{m^{2}} .
$$

In general, there are two ingredients to the conclusion that the higher gradient terms can be neglected in an energy functional: i) $q^{2} \ll \Lambda^{2}$ for typical $q^{2}$ and ii) the coefficient is of order unity [as in Eq. (4)]. A predictive power counting prescription relies on both. For nuclei such as lead, $\left\langle q^{2}\right\rangle$ is small in the interior and is largest in the surface, where the scale is set by the surface thickness $\sigma$. Typically $1 / \sigma \Lambda \approx 1 / 5$ [16]. The power counting analysis in Ref. [16] shows that resolving individual mass terms, which would mean determining two terms in each isoscalar meson propagator, is not possible (that is, only one gradient term is constrained by the fit).

We can test this conclusion directly by varying the heavy meson masses. In Fig. 团, the skin thicknesses of three nuclei are plotted against the isoscalar vector meson mass in a covariant meson model. As the vector mass was varied over a factor of two, the model parameters were adjusted to achieve roughly the same goodness of fit. The corresponding scalar mass is shown on the top axis (note that it varies much less than a factor of two). While strict attention to the $\chi^{2}$ of the fit favors a vector meson mass between 700 and $800 \mathrm{MeV}$, the variation over the entire range is remarkably small. This confirms that the range of the individual mesons is not well determined by the fit to nuclei and that a gradient expansion is more than adequate for this observable.

The concept of optimal parameters for covariant models was introduced in Refs. [17, 45] and further exploited in Ref. [16]. This is a reorganization of the power counting hierarchy by introducing linear combinations of parameters based on the close cancellations in covariant models between scalar and vector contributions. By rewriting in terms of the optimal parameters, the number of parameters actually determined by the data is manifested. For example, the combination:

$$
-\frac{g_{\mathrm{s}}^{2}}{m_{\mathrm{s}}^{2}}+\frac{g_{\mathrm{v}}^{2}}{m_{\mathrm{v}}^{2}}
$$

is much better determined than each of these terms individually. The choice of optimal parameters is not unique and will depend on the quantity to be studied. To identify appropriate optimal parameters for studying masses (gradients) in covariant meson models, we note that the mass terms can be rewritten:

$$
\frac{1}{2}\left(m_{\mathrm{s}}^{2} \phi^{2}-m_{\mathrm{v}}^{2} V_{0}^{2}\right)=\frac{1}{2}\left(m_{\mathrm{s}} \phi+m_{\mathrm{v}} V_{0}\right)\left(m_{\mathrm{s}} \phi-m_{\mathrm{v}} V_{0}\right) \equiv \frac{1}{2} \Phi_{+} \Phi_{-},
$$

which defines the optimal combinations $\Phi_{+}$and $\Phi_{-}$. Since $\Phi_{+} \gg \Phi_{-}$for meson models, if we rewrite the gradient terms using these variables, we find

$$
\frac{1}{2}(\nabla \phi)^{2}-\frac{1}{2}\left(\nabla V_{0}\right)^{2}=\frac{1}{8} \frac{1}{\bar{m}^{2}}\left(\nabla \Phi_{+}\right)^{2}+\mathcal{O}\left(\nabla \Phi_{+} \cdot \nabla \Phi_{-},\left(\nabla \Phi_{-}\right)^{2}\right),
$$




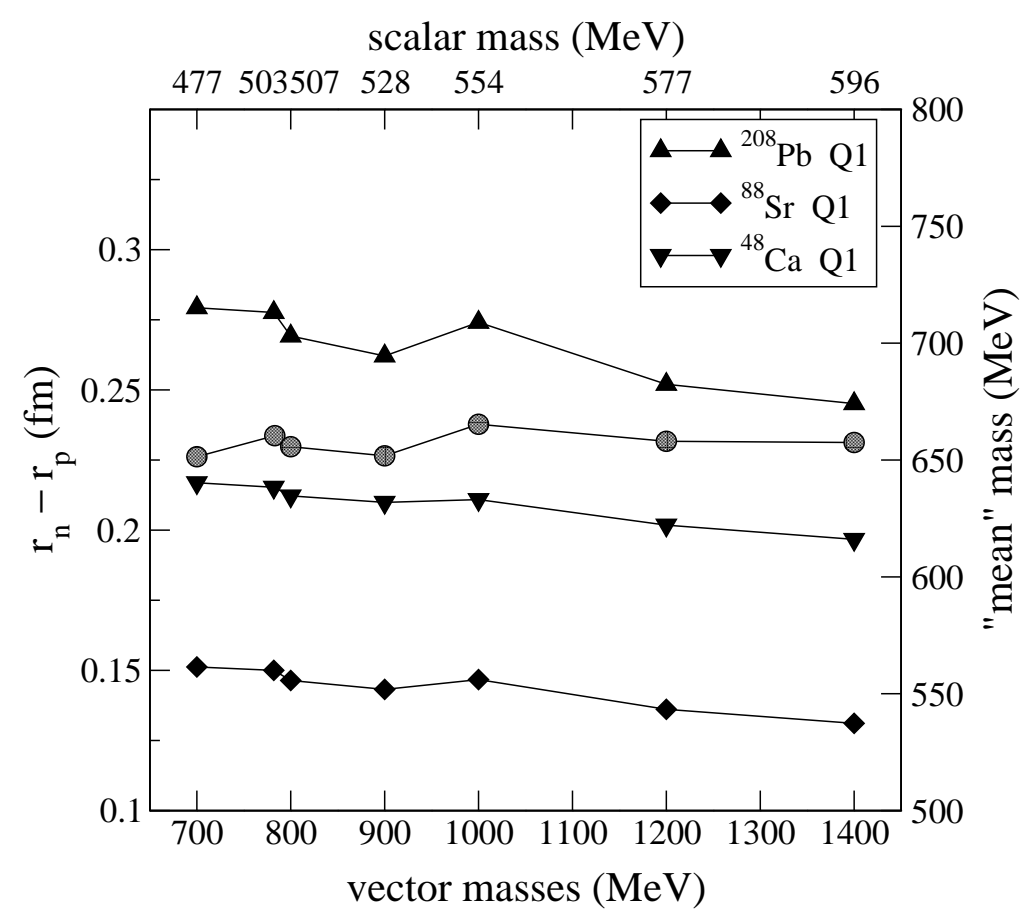

FIG. 4: Neutron skin thickness for three nuclei vs. the isoscalar vector (bottom axis) and scalar (top axis) meson masses in a relativistic mean-field model (Q1) from Ref. [19]. The circles denote the "mean" meson mass (right axis).

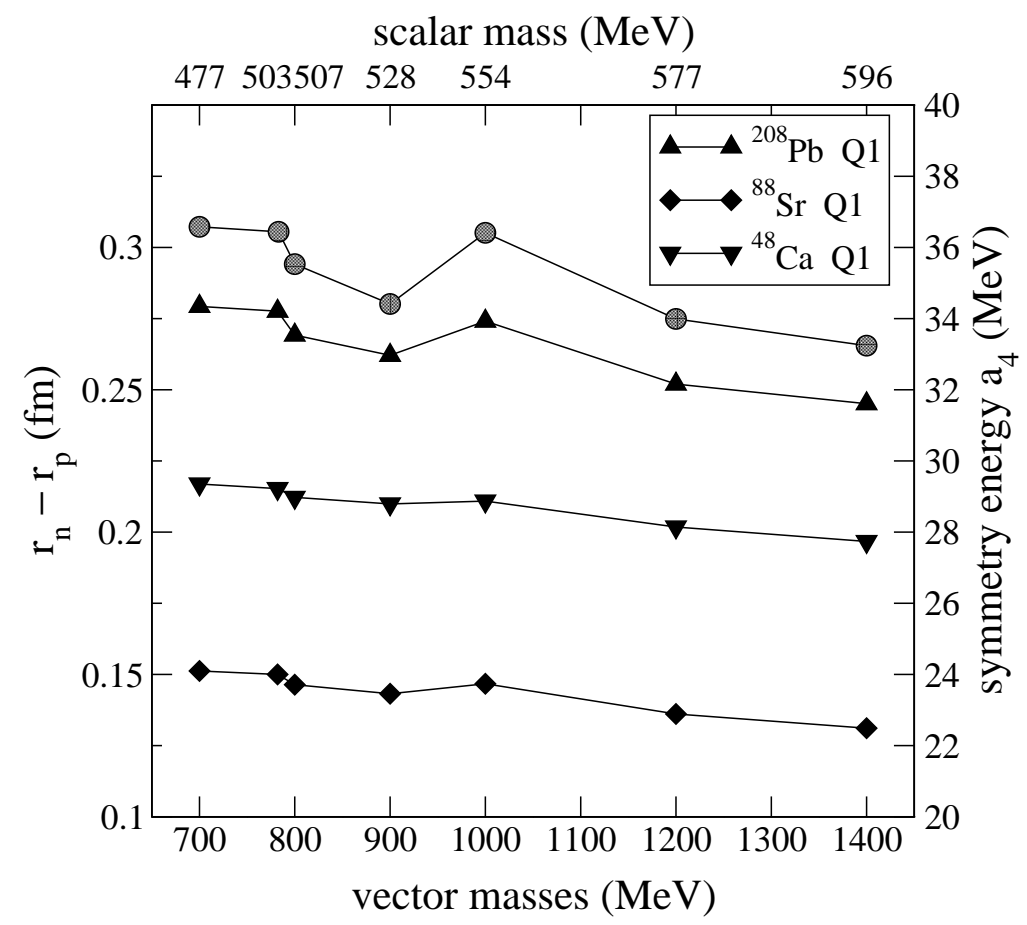

FIG. 5: Neutron skin thickness for three nuclei vs. the isovector vector (bottom axis) and scalar (top axis) meson masses in a relativistic mean-field model (Q1) from Ref. [19]. The circles denote the bulk symmetry energy $a_{4}$ (right axis) in the adjusted parameter set. 
with

$$
\frac{1}{\bar{m}^{2}} \equiv \frac{1}{m_{s}^{2}}-\frac{1}{m_{v}^{2}}
$$

This implies that the "mean" mass $\bar{m}$ is much better determined than either of the individual meson masses. This conclusion is verified in Fig. 4 , in which $\bar{m}$ is plotted as circles (the scale is on the right axis). The mass $\bar{m}$ varies by less than $20 \mathrm{MeV}$ over the entire range of variation of the scalar and vector masses. Thus an (isoscalar) mass scale of about $600 \mathrm{MeV}$ underlies the model; we identify it with the scale $\Lambda$ used in the power counting.

One might argue that the usefulness of optimal parameters imply that a non-covariant EFT is more appropriate. In Refs. [16] and [15] this viewpoint is challenged in detail. Here we simply note that the large underlying scales characteristic of covariant models are the relevant scales in the successful power counting prescription, and that the same scale $\Lambda$ is consistent with power counting in nonrelativistic Skyrme models [18].

If we examine Fig. 1 in more detail, we observe a slight downward trend in the skin thickness, particularly for lead; one might think that this is the residual effect of the meson range. In fact, we can account for this trend completely by considering another plausible candidate for the source of variation in predicted neutron skins: the symmetry energy. We introduce the parameter

$$
\alpha \equiv \frac{N-Z}{A}
$$

and write the semi-empirical mass formula for the energy per particle in nuclear matter at equilibrium as

$$
E / A=-a_{v}+a_{4} \alpha^{2}+a_{s} / A^{1 / 3}+\ldots .
$$

In Fig. 5, the symmetry energy $a_{4}$ for each refit model is plotted as a circle (the scale is on the right axis). The trend in symmetry energy is perfectly correlated with the skin thickness.

Can most of the variation in neutron skin thickness between models be understood in terms of bulk nuclear matter properties, or is the explicit surface symmetry energy also important? In Ref. [24, this issue was investigated by considering a macroscopic "droplettype" model of nuclei that divides the energy functional for the binding energy into an infinite matter piece and the lowest-order gradient terms. Specifically, the binding energy $B(Z, A)$ for $Z$ protons and $A-Z$ neutrons was calculated as

$$
-B(Z, A) \equiv \int d^{3} r E(\rho, \alpha) \rho(r)+\int d^{3} r F_{0}\left(|\nabla \rho|^{2}-\beta\left|\nabla \rho_{3}\right|^{2}\right)+\text { coulomb , }
$$

where $\rho \equiv \rho_{n}(r)+\rho_{p}(r)$ is the baryon density, $\rho_{3} \equiv \rho_{n}(r)-\rho_{p}(r)$ and $\alpha(r) \equiv \rho_{3}(r) / \rho(r)$ is the local ratio. Surface properties such as the neutron skin come from both density dependence in $E(\rho, \alpha)$ and explicit contributions proportional to $F_{0}$ and $\beta$. By fitting this model to nuclear binding energies, the authors of Ref. [24] concluded that the explicit surface symmetry energy (i.e., contributions proportional to $\beta$ ) was of minor importance.

This model is quite compatible with the density functional approach. In fact, we have precisely the form of Eq. (11) with Skyrme and covariant point-coupling energy functionals, and for meson models one can use the meson equations iteratively to get estimates for the parameters $F_{0}$ and $\beta$. We can estimate (and bound) the contribution from the surface symmetry energy by adopting natural values of the associated parameters and then observing the variation in $r_{n}-r_{p}$ as $\beta$ runs over the natural range. The NDA estimate, which applies 


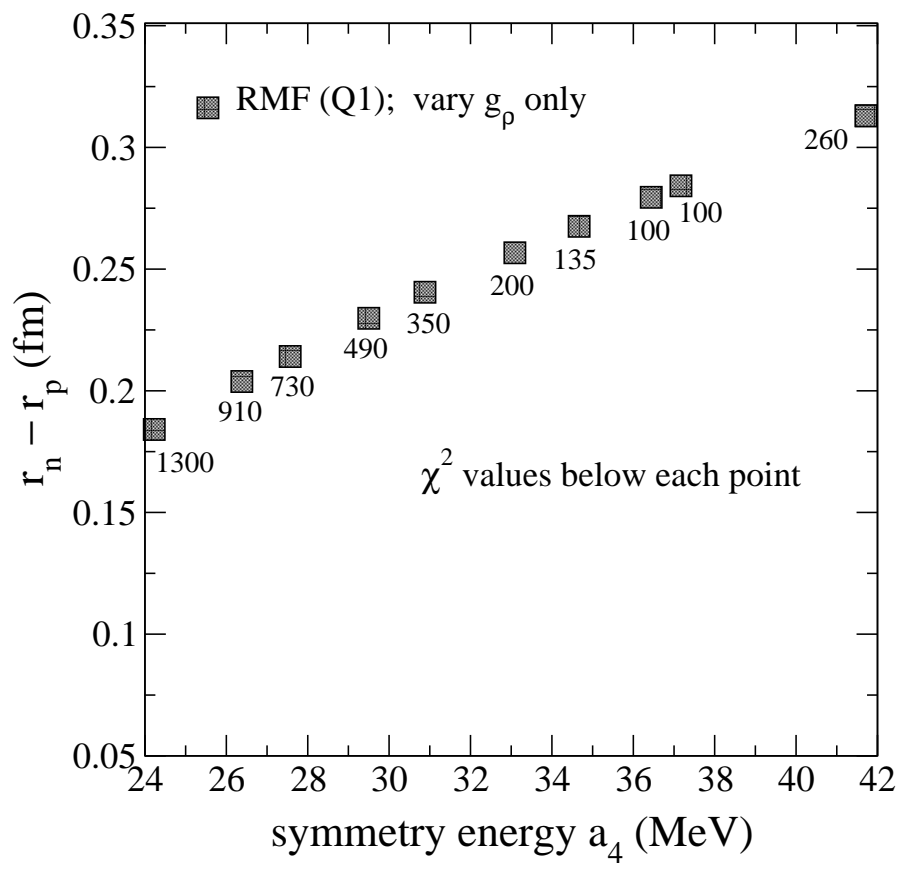

FIG. 6: Neutron skin thickness in ${ }^{208} \mathrm{~Pb}$ vs. symmetry energy $a_{4}$ for a single covariant meson mean-field model. The symmetry energy is varied by changing the rho meson coupling constant, without refitting. The chi-square values are shown with the data points.

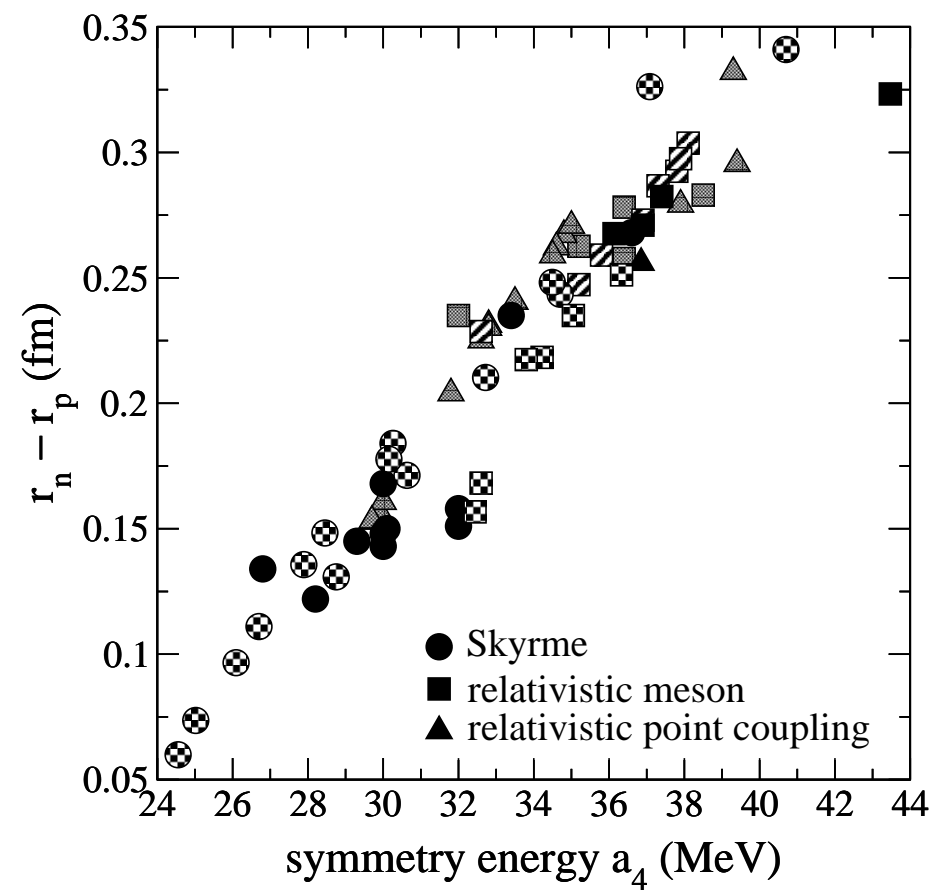

FIG. 7: Neutron skin thickness in ${ }^{208} \mathrm{~Pb}$ vs. symmetry energy $a_{4}$ for a wide variety of mean-field models, as described in the text and in the caption to Fig. 2 . 


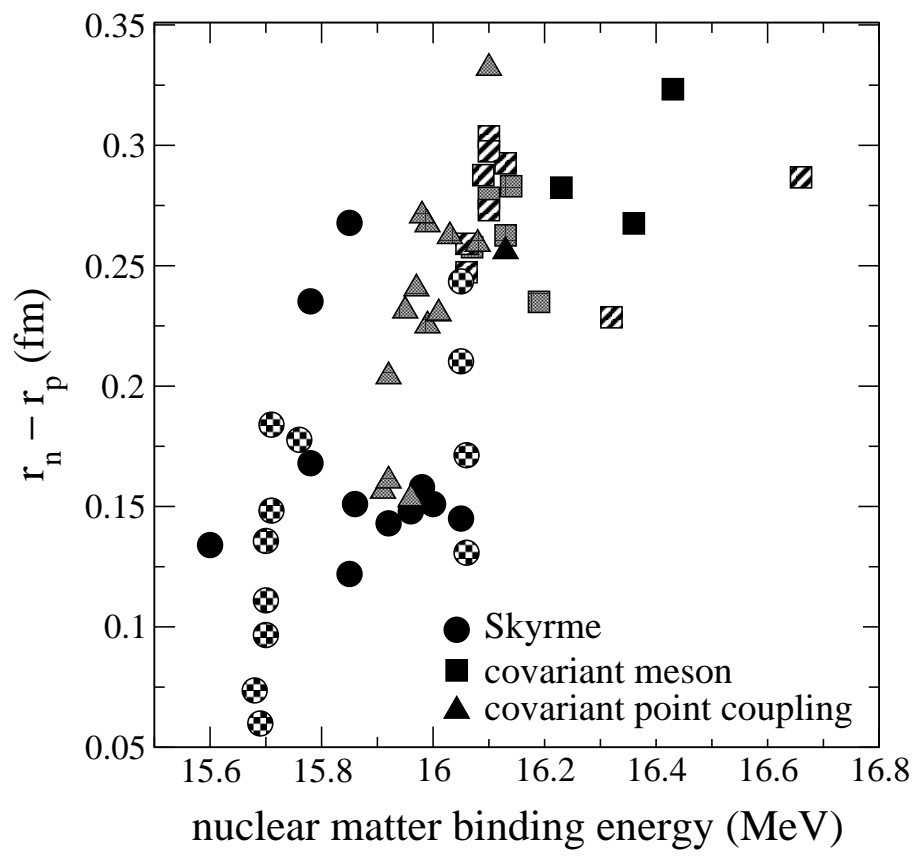

FIG. 8: Neutron skin thickness vs. nuclear matter binding energy for a wide variety of mean-field models, as described in the text and in the caption to Fig. 2.

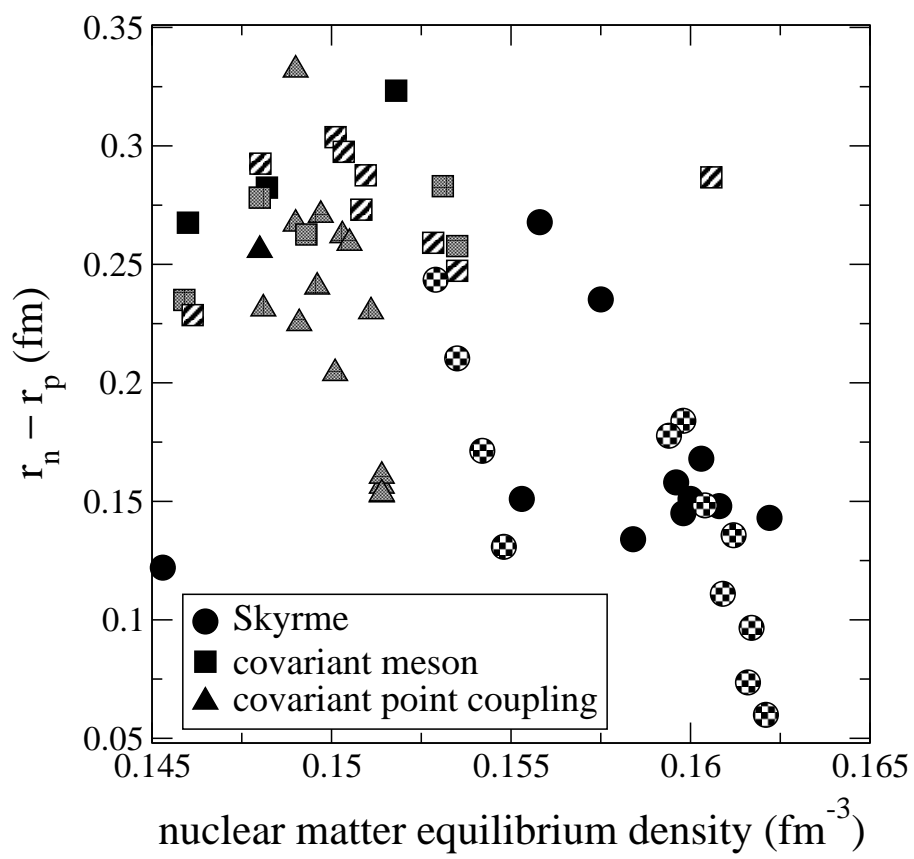

FIG. 9: Neutron skin thickness vs. nuclear matter equilibrium density (in $\mathrm{fm}^{-3}$ ) for a wide variety of mean-field models, as described in the text and in the caption to Fig. 2. 


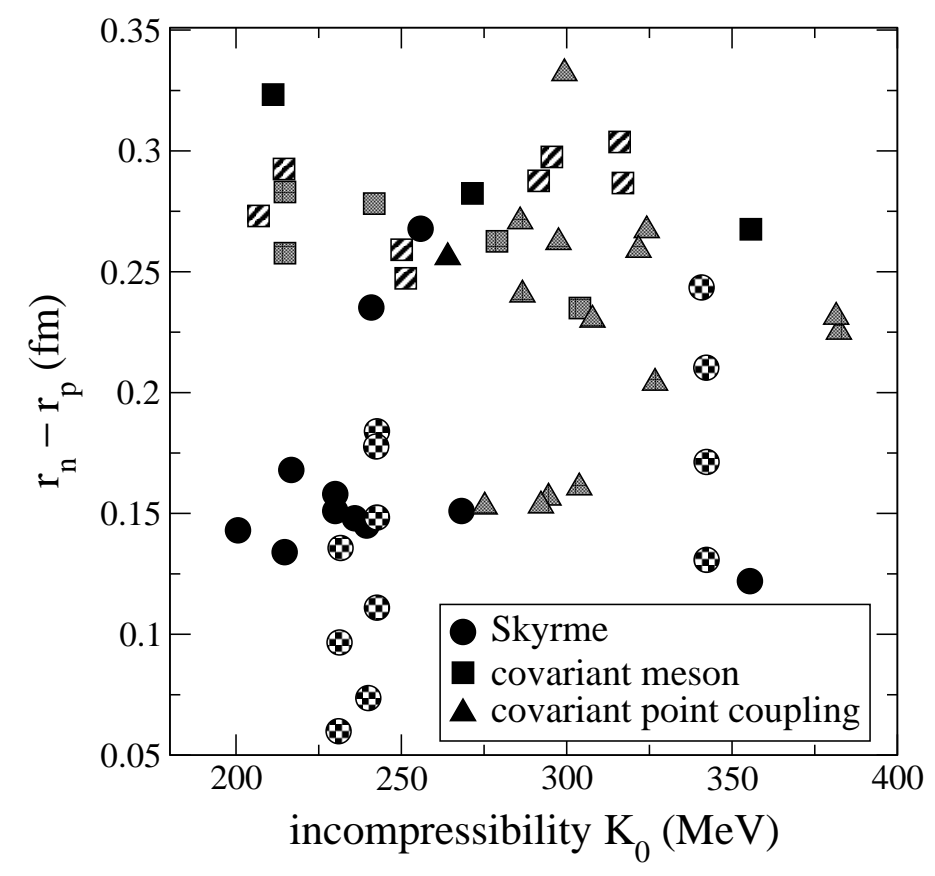

FIG. 10: Neutron skin thickness vs. nuclear matter incompressibility for a wide variety of mean-field models, as described in the text and in the caption to Fig. 2.

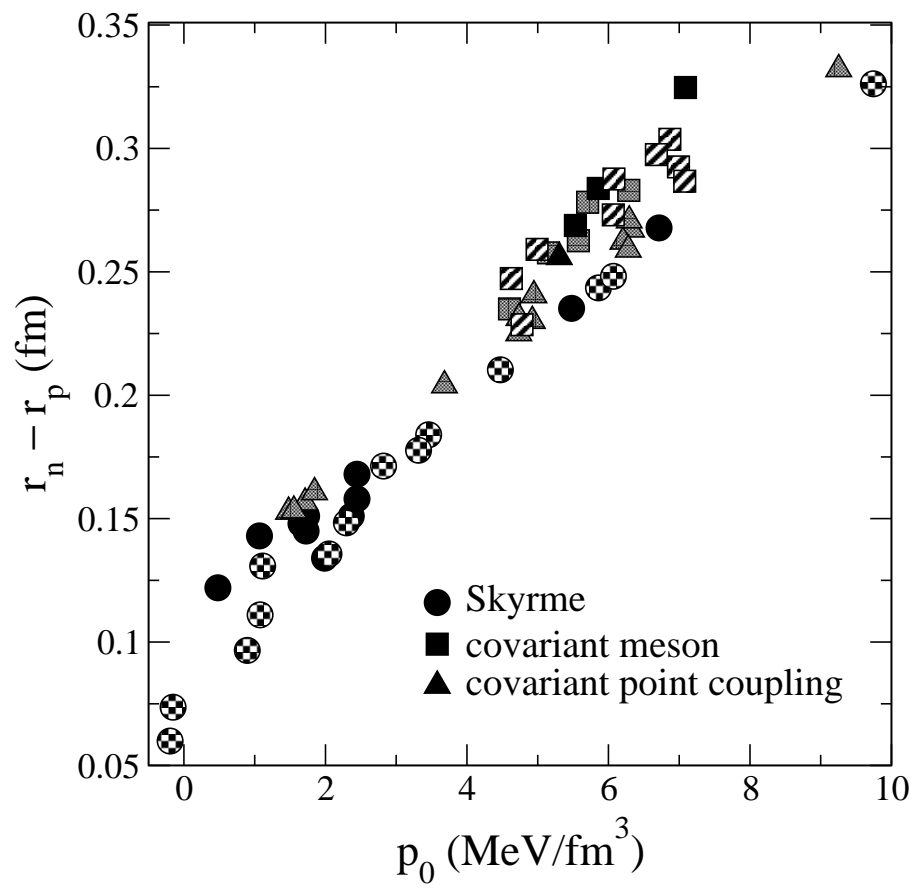

FIG. 11: Neutron skin thickness in ${ }^{208} \mathrm{~Pb}$ vs. linear density dependence of the symmetry energy $p_{0}$ for a wide variety of mean-field models, as described in the text and in the caption to Fig. 2 . 
directly to either Skyrme or covariant point-coupling mean-field models, is

$$
F_{0}\left(|\nabla \rho|^{2}-\beta\left|\nabla \rho_{3}\right|^{2}\right) \sim f_{\pi}^{2} \Lambda^{2}\left[\frac{1}{\Lambda^{2}} \frac{1}{\left(f_{\pi}^{2} \Lambda\right)^{2}}\left\{\mathcal{O}(1)-\frac{1}{4} \alpha^{2} \mathcal{O}(1)\right\}\right] .
$$

Numerical estimates for $\Lambda=600 \mathrm{MeV}$ are $F_{0} \sim(\hbar c)^{5} / f_{\pi}^{2} \Lambda^{2} \sim 75 \mathrm{MeV}-\mathrm{fm}^{5}$ and $\beta \sim \mathcal{O}(1 / 4)$, with the signs undetermined. (The factor of $1 / 4$ originates with the association $\psi^{\dagger}\left(\frac{1}{2} \tau_{3}\right) \psi \leftrightarrow$ $\frac{1}{2} \rho_{3}$.) These values are consistent with all of the Skyrme and point-coupling model fits, which is another verification of naturalness.

Varying $\beta$ between -1 and +1 leads to a change in the neutron skin thickness of less than $0.03 \mathrm{fm}$ for ${ }^{208} \mathrm{~Pb}$, which is small compared to the typical spread between covariant and Skyrme models. Thus the explicit surface dependence of the symmetry energy is not a significant factor. ${ }^{\S}$ This means that we can focus our attention on nuclear matter properties.

In general, the energy per particle of asymmetric matter can be expanded about the equilibrium density $\rho_{0}$ in a Taylor series in $\rho$ and $\alpha$ (we follow the notation of Ref. [60]:

$$
\begin{gathered}
E(\rho, \alpha)=E(\rho, 0)+S_{2}(\rho) \alpha^{2}+S_{4}(\rho) \alpha^{4}+\cdots \quad \alpha \equiv \frac{N-Z}{A} \\
E(\rho, 0)=-a_{v}+\frac{K_{0}}{18 \rho_{0}^{2}}\left(\rho-\rho_{0}\right)^{2}+\cdots \\
S_{2}(\rho)=a_{4}+\frac{p_{0}}{\rho_{0}^{2}}\left(\rho-\rho_{0}\right)+\frac{\Delta K_{0}}{18 \rho_{0}^{2}}\left(\rho-\rho_{0}\right)^{2}+\cdots,
\end{gathered}
$$

which defines the linear density dependence of the symmetry energy, $p_{0}$, and the correction to the incompressibility, $\Delta K_{0}$. These parameters and the isoscalar equilibrium parameters $a_{v}, \rho_{0}$, and $K_{0}$ are our candidates for correlations with the neutron skin. The contribution of $S_{4}(\rho)$ for ${ }^{208} \mathrm{~Pb}$ is very small because of the $\alpha^{4}$ factor and so $S_{4}$ is not constrained in meanfield models, making extrapolations to $\alpha \approx 1$ (neutron matter) quite uncertain (although $S_{2}$ dominates near $\rho_{0}$ in any case). We note that a study using realistic interactions found $S_{4}(\rho)$ to be unimportant even at higher densities [60.

One might try to study the correlations quantitatively by varying appropriate parameters one by one within a single model, as illustrated in Fig. 6. The symmetry energy at equilibrium in a single relativistic meson mean-field model (set Q1 from Ref. [19]) is varied by changing the value of the rho coupling constant $g_{\rho}$ [see Eq. (16)]. It is clear from the figure that the neutron skin is linearly correlated with the symmetry energy. But if instead we plot in Fig. 7 the skin thickness versus $a_{4}$ for a wide range of models, each with a good fit to nuclear properties (binding energies, charge radii, spin-orbit splittings), we see strong correlation again but with a significantly different slope. The problem is that one cannot vary one parameter independently and expect that the fit remains good. The goodnessof-fit value (labeled $\chi^{2}$ ) for each set is shown in Fig. 6, with a value of 100 indicating an acceptable fit. This shows that the quality deteriorates rapidly unless the other parameters are allowed to change. A more sophisticated approach to the correlation analysis during the fitting process would be very helpful in future investigations.

$\S$ There is a loophole here: If $\beta$ is actually determined by long-range pion physics, the scale of the gradient might be set by $m_{\pi} \ll \Lambda$, which would mean $\beta$ could be much larger than unity. 


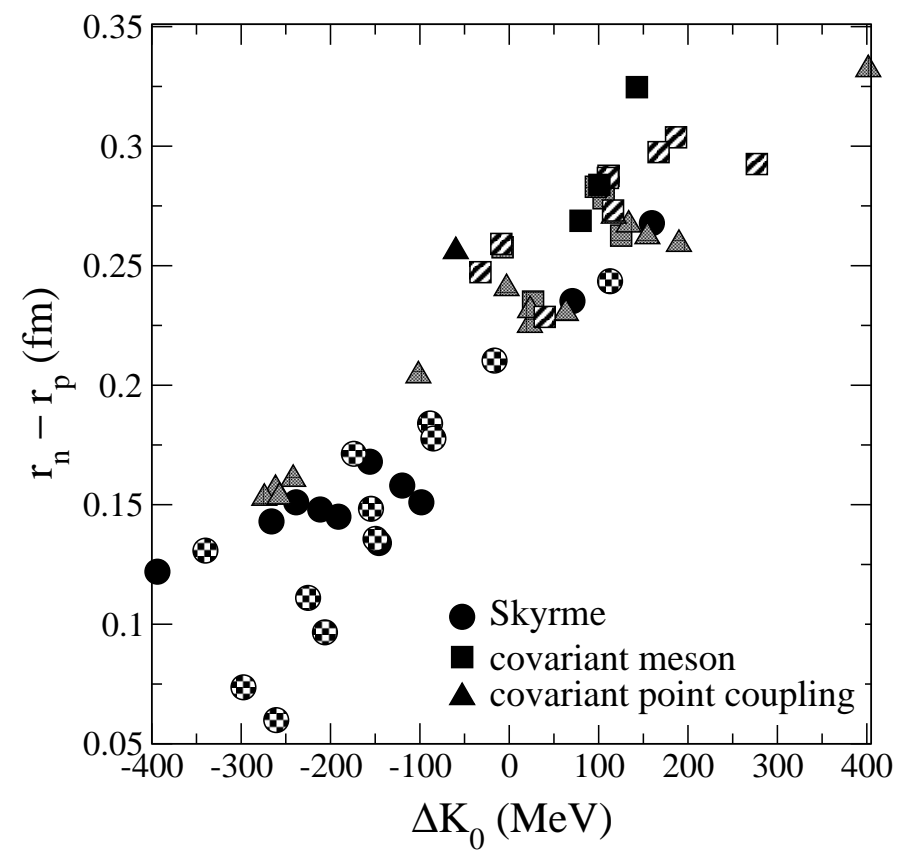

FIG. 12: Neutron skin thickness in ${ }^{208} \mathrm{~Pb}$ vs. $\Delta K$ [see Eq. (15)] for a wide variety of mean-field models, as described in the text and in the caption to Fig. 2 .

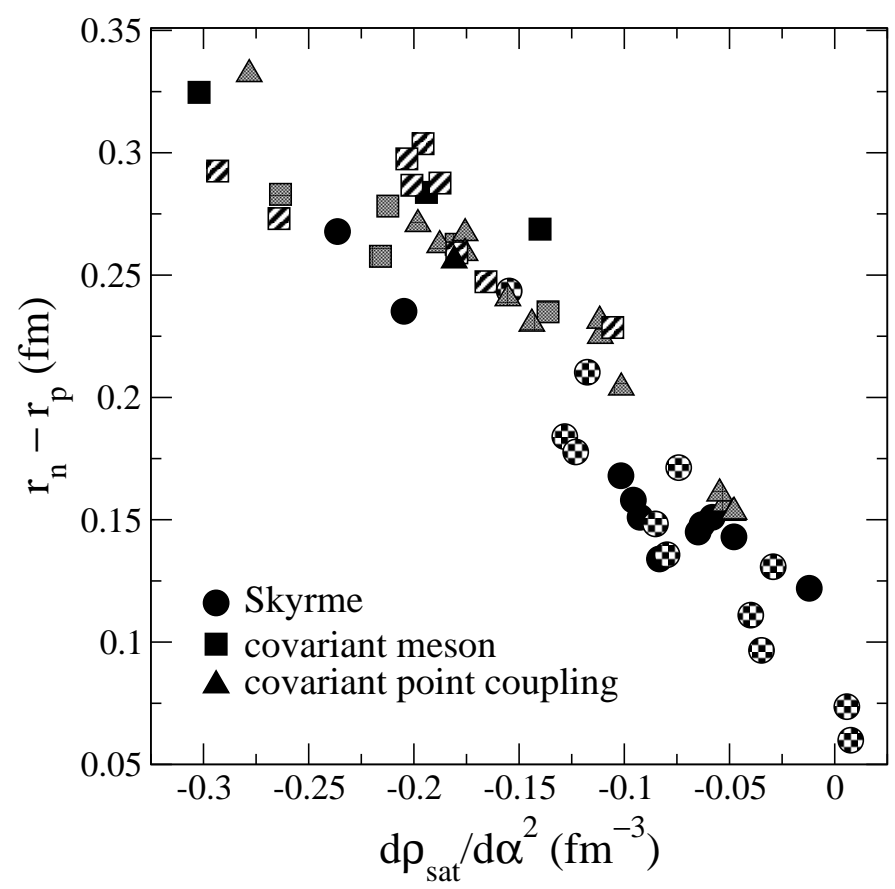

FIG. 13: Neutron skin thickness in ${ }^{208} \mathrm{~Pb}$ vs. $d \rho_{\text {sat }} / d \alpha^{2}$ for a wide variety of mean-field models, as described in the text and in the caption to Fig. 2. 
In Figs. 8, 9, and 10, the correlations of the skin thickness with the symmetric nuclear matter $(\alpha=0)$ binding energy, equilibrium density, and incompressibility are shown. The coarse observation is that there are no dramatic correlations between these quantities individually and the skin thickness. At a more fine-grained level one can note:

- The binding energy of Skyrme models is systematically lower than for covariant models, although the difference is small and there is significant overlap.

- The equilibrium density of Skyrme models is systematically larger than for covariant models. This has long been observed. There is a very rough correlation with skin thickness.

- The incompressibility seems largely uncorrelated with the skin thickness and there are no clear patterns between different types of models.

Overall, we find no signature that the physics determining these quantities directly determines the skin thickness. Secondary correlations are expected simply from the requirement that a good fit is always obtained.

In Figs. 7, 11, and 12, we show the correlations between skin thickness and the individual parameters describing the symmetry energy $S_{2}(\rho)$. In each case the correlation is very strong and approximately linear. The spread for $p_{0}$, in particular, is remarkably small and there is no significant separation of the different models: to good approximation all covariant and Skyrme models lie on the same line. A clear corollary to the correlations of $a_{4}, p_{0}$, and $\Delta K_{0}$ to the neutron skin thickness is that these parameters are also highly correlated with each other.

\section{DISCUSSION}

Qualitatively, the neutron skin is determined from the energy balance sought for $N \neq Z$ between the extremes of equal proton and neutron radii but different densities, and equal densities but a sizable neutron skin region. Thus, the cost in energy is determined in large part by the density dependence of the symmetry energy, $S_{2}(\rho)$ [24]. In addition, the Coulomb repulsion favors larger proton radii, which reduces the size of the neutron skin in lead approximately $0.1 \mathrm{fm}$ relative to the radii with Coulomb turned off, with only a small residual dependence on the symmetry energy. (This result was obtained by comparing radii with and without the Coulomb interaction for each model.) A simple density-expansion analysis based on these ideas made by Oyamatsu et al. [24] identifies as a key parameter the shift in saturation point with the asymmetry, $d \rho_{\text {sat }} / d \alpha^{2}=-9 p_{0} / K_{0}$. The correlation of the neutron skin with $d \rho_{\text {sat }} / d \alpha^{2}$ is shown in Fig. 13$]$ and is strong, although there is greater spread than with $p_{0}$ alone.

An explanation of the strong correlation in mean-field models between the parameters of $S_{2}(\rho)$ has been offered by Horowitz [61]. There is not enough resolution in the binding energy systematics of finite nuclei to fix $S_{2}(\rho)$ for a range of densities. In fact, as discussed earlier, only one quantity is resolved, so one determines the symmetry energy only at an average density $\langle\rho\rangle$ for nuclei. (For lead we find $\langle\rho\rangle \approx 0.11 \mathrm{fm}^{-3}$.) Consequently there are many combinations of $a_{4}, p_{0}$, and $\Delta K_{0}$ (and $\rho_{0}$ ) that reproduce this value. This conjecture is supported by the observation that the spread of $S_{2}(\langle\rho\rangle)$ values among the models used in the figures is several times smaller than the spread of $S_{2}\left(\rho_{0}\right)$ values, as shown in Fig. 14. 


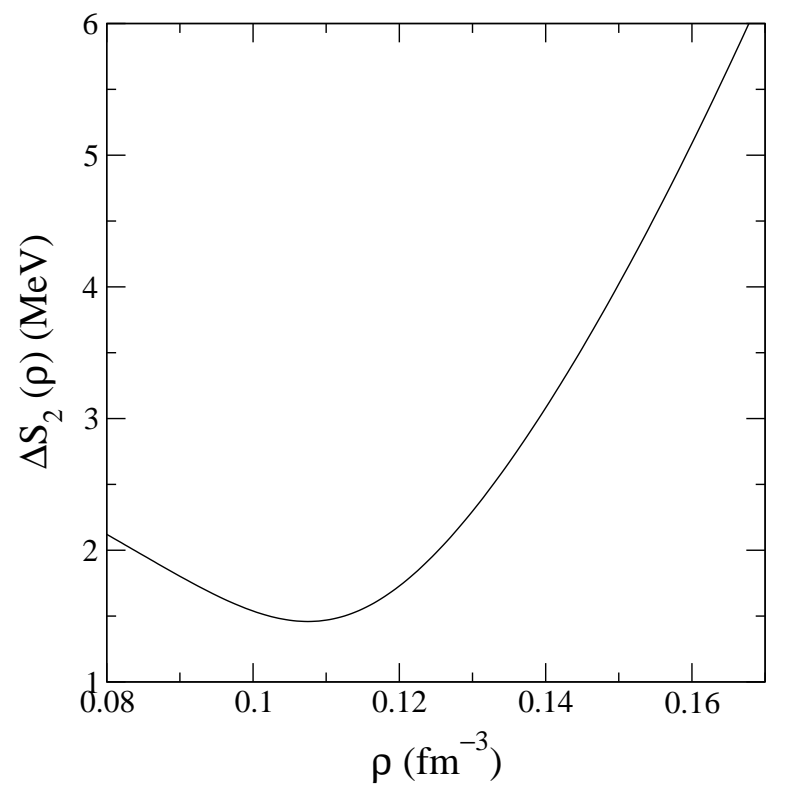

FIG. 14: The standard deviation of $S_{2}(\rho)$ values for all of the mean-field models as a function of $\rho$.

The standard parameterization of energy functionals for covariant meson models leads to an enhanced symmetry energy because the kinetic energy piece is large (since $M^{*}$ is reduced from $M$ ) and the contribution from the $\rho$ meson mean field is positive definite. Specifically, $a_{4}$ is given by

$$
a_{4}=\frac{1}{6} \frac{k_{\mathrm{F}}^{2}}{\left(k_{\mathrm{F}}^{2}+M^{* 2}\right)^{1 / 2}}+\frac{g_{\rho}^{2}}{8 m_{\rho}^{2}} \rho_{0},
$$

where $g_{\rho}$ is the coupling, $m_{\rho}$ is the $\rho$ mass parameter, $k_{\mathrm{F}}$ is the Fermi momentum, and $M^{*}$ is the effective nucleon mass [35]. In addition, $p_{0}$ scales with $a_{4}$ (e.g., the $\rho$ contribution to $p_{0}$ is $\left.g_{\rho}^{2} \rho_{0}^{2} / 8 m_{\rho}^{2}\right)$. If fits to nuclei determine the symmetry energy at some averaged density less than equilibrium density $S_{2}(\langle\rho\rangle)$, this implies that both $a_{4}$ and $p_{0}$ will be large (i.e., a large density dependence). In fact, typical values for $p_{0}$ are around $6 \mathrm{MeV} / \mathrm{fm}^{3}$, compared to about $2 \mathrm{MeV} / \mathrm{fm}^{3}$ for most standard Skyrme models, while the corresponding values for $a_{4}$ are around $36 \mathrm{MeV}$ and $30 \mathrm{MeV}$.

Given this association, we can ask: what should $p_{0}$ be? Engvik et al. compared calculations of asymmetric matter using the best-available nonrelativistic potentials and many-body calculations [62]. From the slopes of the curves given in Ref. [62], one can determine $p_{0}$ (up to a factor of $\rho_{0}^{2}$ ). One finds $p_{0} \approx 2 \mathrm{MeV} / \mathrm{fm}^{3}$ with only about a $10 \%$ spread. [Note: there is some ambiguity in the choice of $\rho_{0}^{2}$ to compare with mean-field models; if a larger value associated with some Skyrme models was used, then $p_{0}$ would be 10-30\% higher.] Two Dirac-Brueckner studies of asymmetric nuclear matter 60, 63 yield somewhat higher numbers, $p_{0} \approx 3 \mathrm{MeV} / \mathrm{fm}^{3}$. However, both sets of values are significantly less than those from the standard covariant mean-field meson models (i.e., the solid black squares in Fig. 11). Generalized meson models can be forced to have lower $p_{0}$ (by adding a low neutron radius to the fit observables) but sets with $p_{0}$ lower than $5 \mathrm{MeV} / \mathrm{fm}^{3}$ are only achieved if the additional isovector parameter $\left(\eta_{\rho}\right.$ from Ref. [19]) becomes highly unnatural.

Generalized point-coupling models [17], which feature higher-order terms not included in conventional covariant models, were found with lower values of $p_{0}$ and good fits (see the 
shaded triangles in Fig. 11). However, this lower value arises from cancellations between two orders in the density expansion for the isovector contribution, which again violates the principle of naturalness. In particular, the contribution to $p_{0}$ analogous to the $\rho$ meson contribution is proportional to $\widetilde{\zeta}_{2}+\frac{3}{2} \widetilde{\eta}_{\rho} \widetilde{\rho}_{0}$, where $\widetilde{\rho}_{0} \approx 1 / 5$. By choosing $\widetilde{\eta}_{\rho} \approx-10 / 3$, one can cancel the leading contribution for natural $\widetilde{\zeta}_{2} \approx 1$. In fact, one of the sets has $\widetilde{\eta}_{\rho}=$ -3.245 . We do not have a physical reason for such a fine tuning between different orders and conjecture that it may reflect instead limitations in the isovector contribution to the energy functional.

The conclusion is therefore that we need to revisit isovector physics in covariant models. There are many possible deficiencies to explore:

- The functional is incomplete. For example, isovector scalar contributions (the " $\delta$ " meson) may be important for isovector properties. This possibility has been recently considered in Ref. [46].

- The functional is truncated too severely. The higher-order terms discussed above are an example of what could be added.

- Long-range physics must be explicitly included, as expected in an EFT treatment. One natural class of contributions is from the pion. As noted above, explicit pions have not been necessary because the isoscalar part is shorter-ranged ( $\sigma$ " physics) and the isovector part is poorly resolved. However, power counting implies that pion contributions should be included. In addition, many-body long-range correlations (e.g., RPA correlations) may be needed.

- The one-loop form of the functional is not sufficiently general. For example, one will obtain nonanalytic terms in the density from the long-range contributions.

We seek a systematic approach to incorporating this physics into our descriptions of medium and heavy nuclei. Furthermore, a connection to underlying forces is desirable to help constrain the functional.

A key question is whether mean-field models can be made more systematic without drastically increasing the computational burden. A great appeal of mean-field models is the ease with which they can be used to calculate medium to heavy nuclei. The basic calculational procedure is the same for a covariant Hartree or nonrelativistic Skyrme interaction. The models can all be formulated in terms of an energy functional, whose minimization with respect to single-particle orbitals yields a single-particle "potential" functional. This potential is used in turn to calculate the orbitals.

The generic procedure is quite simple. To solve for the ground-state bulk properties of a nucleus with $A=Z+N$ nucleons,

1. guess a set of initial density/current profiles $\{\rho(\mathbf{x})\}$ (this notation denotes the baryon and isospin densities, plus any other spin or scalar densities); most can be set to zero or initialized with a Fermi shape with appropriate parameters;

2. evaluate a functional of the $\{\rho(\mathbf{x})\}$, yielding a local single-particle potential $V_{s}(\mathbf{x})$;

3. solve the Dirac or Schrödinger equation for the lowest $A$ eigenvalues and eigenfunctions $\left\{\epsilon_{\alpha}, \psi_{\alpha}\right\}$ :

$$
\left[-i \boldsymbol{\alpha} \cdot \boldsymbol{\nabla}+\beta M+\beta V_{s}(\mathbf{x})\right] \psi_{\alpha}(\mathbf{x})=\epsilon_{\alpha} \psi_{\alpha}(\mathbf{x})
$$


or

$$
\left[-\frac{\nabla^{2}}{2 M}+V_{s}(\mathbf{x})\right] \psi_{\alpha}(\mathbf{x})=\epsilon_{\alpha} \psi_{\alpha}(\mathbf{x})
$$

4. compute new densities, e.g.,

$$
\rho(\mathbf{x})=\sum_{\alpha=1}^{A}\left|\psi_{\alpha}(\mathbf{x})\right|^{2}
$$

and other observables (which are functionals of $\left\{\epsilon_{\alpha}, \psi_{\alpha}\right\}$ );

5. repeat steps 2.-4. until the changes from iteration to iteration are acceptably small, i.e., until the solution is self-consistent.

This procedure is straightforward to implement numerically. Dealing with deformed nuclei and open shells introduces some complications (e.g., pairing), but these do not qualitatively complicate the calculations. There are two key reasons for the simplicity:

- the energy functional is universal, in the sense that the same functional is used for all nuclei, with the same set of parameters;

- the single-particle functional, which is derived from the functional derivative of the energy functional with respect to $\rho(\mathbf{x})$ is local (at least when pairing is treated most simply) and is easily evaluated in terms of the eigenvalues and eigenfunctions.

Once the functional is established, each subsequent finite nucleus calculation is almost trivial. Thus it is certainly desirable to maintain this mean-field calculational procedure.

In Ref. [6], it is argued that the mean-field approximation for meson-ranged interactions cannot be valid at ordinary densities, because the inverse Compton wavelengths $\mu$ of the mesons times the mean interparticle spacing is much less than unity. The authors go on to state that "The RMF approximation can be based on effective values of the coupling constants that take into account the correlation effects. However, these coupling constants then have a density dependence, and a microscopic theory is needed to calculate them." The second objection is misleading, since density dependent couplings are not necessary; all of the density dependence can be incorporated in the functional, if made sufficiently general. The correspondence between models with explicitly density dependent couplings and more general functionals can be made through field redefinitions [15]. Matching to a microscopic theory may be a desirable way to determine the parameters, but it is not necessary. Instead, one can match to finite density observables, which is especially needed for calculating heavy nuclei.

To address the first objection, we propose a merger of Density Functional Theory (DFT) and Effective Field Theory (EFT). Kohn-Sham DFT is a framework that looks just like the simple relativistic Hartree calculations that are so well adapted to calculating finite systems, but one which can include all short-range and long-range correlations [9]. That is, the solution framework described above can accommodate the most general description of the ground state within the context of density functional theory, and the Kohn-Sham scheme in particular. The implication is that conventional mean-field approaches provide reliable descriptions of bulk properties not because the mean-field approximation is good but because they accurately approximate exact density functionals. This claim is supported by the work of $\mathrm{Hu}$, who showed that Dirac-Brueckner-Hartree-Fock at the two hole-line level, 
when matched to a generalized EFT-based functional, is reproduced with natural coefficients 64.

Therefore, we interpret the "mean-field" functionals as just particular generalized gradient approximations to complete Kohn-Sham density functionals [13, 14]. (Skyrme may be considered more general in this regard.) Rather than starting from the underlying free-space interaction, one expands the functional as a series expansion in density and momentum, and then fits directly to experimental data. This approach can miss (long-range) non-analytic or nonlocal contributions, such as explicit exchange ("Fock") terms (e.g., from pions). The role of the EFT framework is to add these systematically while maintaining symmetries and conservation laws.

From a Green's function perspective, the mean-field models follow from the Hartree (relativistic) or Hartree-Fock (nonrelativistic) approximation to the nucleon self-energy, with an effective interaction (e.g., an approximation to the G-matrix). In practice, the nonrelativistic Skyrme interaction uses zero-range ("point couplings"), which means that the energy functional has the same form as for a Hartree calculation (one can make a Fierz rearrangement of the interaction to derive the correspondence). The Hartree self-energy in coordinate-space is static and local (i.e., a function of $\mathbf{x}$ only).

In contrast, the general self-energy is non-local in space and is frequency dependent. This would seem to imply that the correlation effects that go beyond the Hartree approximation could never be systematically included in the calculational framework described above; one could only incorporate averaged effects using effective interactions. Since the simplicity of the mean-field approach depends heavily on the local, static nature of the single-particle potential, it would appear that systematic improvement is not possible. The answer is that we don't need to calculate the general self-energy. Instead we use the Kohn-Sham DFT potential $V_{s}(\mathbf{x})$, which is static and local, even though it can include all correlations.

There is a danger that evaluating the single-particle functional to find $V_{s}(\mathbf{x})$ will become too great a computational burden when going beyond the current mean-field models. So developing reliable gradient expansions will be essential. The surprising experience from Coulomb systems that LDA plus generalized gradient expansions work extremely well needs to be understood in this context. In particular, it does not rule out long-range physics being incorporated in the same form as existing functionals, although contributions nonanalytic in the densities should be expected. For example, the exchange contribution from a long-range (i.e., effectively massless) particle will have a $\rho^{4 / 3}$ contribution to the energy density. Thus, an EFT treatment of the pion expanded around the chiral limit should contribute a term like this. Work is in progress to cast Kohn-Sham DFT into an effective action formalism for composite operators 65.

We could imagine constructing covariant models more like Skyrme models, by including terms like $\rho_{s}^{\delta}$ and $\rho_{B}^{\gamma}$, with fractional $\delta$ and $\gamma$ determined phenomenologically. But the construction from a Lagrangian has been an important guide to maintaining covariance and building a conserving approximation. In addition, the connection to free space scattering is much closer than in the nonrelativistic case since self-energies are dominated by meanfield components (Hartree dominance), so corrections are smaller. Therefore, we advocate deriving a DFT expansion from an EFT Lagrangian, or at least to establish constraints on the analytic structure.

Finally, we return to the issue of point nuclear densities. Single-nucleon structure will automatically be included in energy functionals fit to data. Since point proton and neutron radii are not observables (no probe couples to them) they are at best auxiliary quantities. 
This is not a major issue for properties such as the charge density alone, since the nuclear structure is mostly isoscalar while the single-nucleon form factors are mostly isovector and so factorization is a good starting point. But considering $r_{n}-r_{p}$ heightens sensitivity to the isovector form factor. In the future, one should actually couple to electroweak currents to avoid the model dependence inherent in defining the point densities. One of the strengths of an EFT is knowing how to couple to external currents, which may involve identifying new constants (low-energy constants).

\section{SUMMARY}

We have examined the predictions by mean-field models of neutron skin thicknesses, which are highly correlated with the neutron radius. We find that the skin thickness is closely related to the density dependence of the symmetry energy, independent of the type of mean field model. In particular, the linear density coefficient $p_{0}$ exhibits a remarkably good linear correspondence. (However, the strong correlations between parameters preclude isolating the effects of a single parameter.) This implies that it is a general feature of the energy functional. Our conclusion is in accord with other analyses in the recent literature [22, 23, 24].

An accurate extraction of the radius from experiment, for even a single nucleus, would provide a new and valuable constraint on the energy functional. Current analyses of proton scattering are consistent with a skin thickness in lead of order 0.1-0.2 fm [66, 67], which would imply $1 \mathrm{MeV} / \mathrm{fm}^{3}<p_{0}<4 \mathrm{MeV} / \mathrm{fm}^{3}$, and recent analyses of antiprotonic atom data also lie in that range [68]. Calculations of uniform matter with realistic potentials show a narrower range of $2 \mathrm{MeV} / \mathrm{fm}^{3}<p_{0}<3 \mathrm{MeV} / \mathrm{fm}^{3}$, with Dirac-Brueckner calculations at the high end and nonrelativistic calculations at the low end. If these conclusions are validated, it means that the uncertainty in neutron radii may be much smaller than implied by comparisons of the current "best fit" calculations.

However, the commonly used mean-field functionals are not equally flexible in accommodating different values of $p_{0}$. In particular, covariant meson models have significantly larger values of $p_{0}$ than found in most Skyrme parametrizations, such as the recent set of Brown [1]. Calculations of uniform matter with realistic potentials imply that $p_{0}$ is close to that of nonrelativistic Skyrme models while it is significantly overestimated in the most widely used relativistic mean-field models. It is not surprising that this does not preclude a good fit to the best measured properties of finite nuclei, given the limited constraint from these properties on purely isovector parameters. It is not clear whether the tendency for Skyrme models to have lower $p_{0}$ is a consequence of the form of truncation or is slightly favored by the fits to other observables. In Ref. [1], additional information, which corresponds to fixing $p_{0}$, was used to constrain one of the isovector parameters.

Such comparisons between nonrelativistic and covariant models might be used to argue that one approach is more "correct" than another. However, the EFT perspective changes the context of the discussion from correctness to efficiency and the effects of truncation. The most commonly used covariant parametrization appears to be deficient because it does not allow lower $p_{0}$ with natural parameters, but the conclusion should be that the functional must be improved. Power counting suggests several avenues: a more complete set of operators at the lower orders (e.g., add an isovector scalar meson or density), higher order contributions (density corrections to rho meson exchange), pion and other long-range contributions. Additional isovector observables, such as binding energies of more asym- 
metric nuclei and observables from collective excitations will be needed to constrain new parameters. The question is: how can we incorporate this physics to improve the covariant functionals (and nonrelativistic functionals) systematically? We propose developing density functional theory in an EFT framework, using effective action techniques [65].

\section{Acknowledgments}

We thank B. Clark, H.-W. Hammer, and B. Serot for useful comments. This work was supported in part by the National Science Foundation under Grant Nos. PHY-9800964 and PHY-0098645.

[1] B. A. Brown, Phys. Rev. C 58 (1998) 220, and references therein.

[2] P. Ring, Prog. Part. Nucl. Phys. 37 (1996) 193, and references therein.

[3] C. J. Horowitz, S. J. Pollock, P. A. Souder, and R. Michaels, Phys. Rev. C 63 (2001) 025501.

[4] C. J. Horowitz and J. Piekarewicz, Phys. Rev. Lett. 86 (2001) 5647.

[5] Report of the "Nuclear Structure and Astrophysics Town Meeting," Oakland, CA, November, 2000, http://snohp1.lbl.gov/ lpr2000/oakland_wp.pdf.

[6] A. Akmal, V. R. Pandharipande, and D. G. Ravenhall, Phys. Rev. C 58 (1998) 1804.

[7] W. Kohn and L. J. Sham, Phys. Rev. A140 (1965) 1133.

[8] R. M. Dreizler and E. K. U. Gross, Density Functional Theory (Springer, Berlin, 1990).

[9] N. Argaman and G. Makov, Am. J. Phys. 68 (2000) 69.

[10] M. Brack, Helv. Phys. Acta 58 (1985) 715.

[11] R. N. Schmid, E. Engel, and R. M. Dreizler, Phys. Rev. C 52 (1995) 164.

[12] R. N. Schmid, E. Engel, and R. M. Dreizler, Phys. Rev. C 52 (1995) 2804.

[13] J. P. Perdew, S. Kurth, A. Zupan, and P. Blaha, Phys. Rev. Lett. 82 (1999) 2544.

[14] J. P. Perdew, K. Burke, and M. Ernzerhof, Phys. Rev. Lett. 77 (1996) 3865; 78 (1997) 1396(E).

[15] R. J. Furnstahl and B. D. Serot, Comm. Nucl. Part. Phys. 2 (2000) A23.

[16] R. J. Furnstahl and B. D. Serot, Nucl. Phys. A671 (2000) 447.

[17] J. J. Rusnak and R. J. Furnstahl, Nucl. Phys. A627 (1997) 495.

[18] R. J. Furnstahl and J. C. Hackworth, Phys. Rev. C 56 (1997) 2875.

[19] R. J. Furnstahl, B. D. Serot, and H.-B. Tang, Nucl. Phys. A615 (1997) 441; (E) A640 (1998) 505 .

[20] R. J. Furnstahl, C. E. Price, and G. E. Walker, Phys. Rev. C 36 (1987) 2590.

[21] R. J. Furnstahl, J. J. Rusnak, and B. D. Serot, Nucl. Phys. A632 (1998) 607.

[22] B. A. Brown, Phys. Rev. Lett. 85 (2000) 5296.

[23] S. Typel and B. A. Brown, Phys. Rev. C 64 (2001) 027302.

[24] K. Oyamatsu, I. Tanihata, Y. Sugahara, K. Sumiyoshi, and H. Toki, Nucl. Phys. A634 (1998) 3.

[25] A. R. Bodmer, Nucl. Phys. 17 (1960) 388.

[26] B. Q. Chen, Z. Y. Ma, F. Grummer, and S. Krewald, Phys. Lett. B 455 (1999) 13.

[27] D. Vretenar, G. A. Lalazissis, and P. Ring, Phys. Rev. C 62 (2000) 045502.

[28] Z. Patyk et al., Phys. Rev. C 59 (1999) 704. 
[29] S. Mizutori, J. Dobaczewski, G. A. Lalazissis, W. Nazarewicz, and P.-G. Reinhard, Phys. Rev. C 61 (2000) 044326.

[30] J. Dobaczewski, Acta Phys. Polon. B 30 (1999) 1647 arXiv:nucl-th/9901036.

[31] S. Krewald, V. Klemt, J. Speth, and A. Faessler, Nucl. Phys. A281 (1977) 166.

[32] K. Pomorski et al., Nucl. Phys. A624 (1997) 349.

[33] J. Friedrich and P.-G. Reinhard, Phys. Rev. C 33 (1986) 335.

[34] E. Chabanat, P. Bonche, P. Haensel, J. Meyer, and R. Schaeffer, Nucl. Phys. A627 (1997) 710 .

[35] B. D. Serot and J. D. Walecka, Adv. Nucl. Phys. 16 (1986) 1.

[36] B. D. Serot, Rep. Prog. Phys. 55 (1992) 1855.

[37] B. D. Serot and J. D. Walecka, Int. J. Mod. Phys. E 6 (1997) 515, and references therein.

[38] M. Rufa, P.-G. Reinhard, J. A. Maruhn, W. Greiner, and M. R. Strayer, Phys. Rev. C 38 (1988) 390.

[39] G. A. Lalazissis, J. König, and P. Ring, Phys. Rev. C 55 (1997) 540.

[40] B. A. Nikolaus, T. Hoch, D. G. Madland, Phys. Rev. C 56 (1997) 177.

[41] J. W. Negele, Phys. Rev. C 1 (1970) 1260.

[42] J. W. Negele and D. Vautherin, Phys. Rev. C 5 (1972) 1472.

[43] R. J. Furnstahl and B. D. Serot, Nucl. Phys. A673 (2000) 298.

[44] J. L. Forest, V. R. Pandharipande and J. L. Friar, Phys. Rev. C 52, 568 (1995).

[45] J. J. Rusnak, Ph.D. thesis, 1997.

[46] B. Liu, V. Greco, V. Baran, M. Colonna, and M. DiToro, arXiv:nucl-th/0112034.

[47] T. Burvenich, D. G. Madland, J. A. Maruhn and P. G. Reinhard, arXiv:nucl-th/0111012.

[48] G. P. Lepage, "What is Renormalization?", in From Actions to Answers (TASI-89), edited by T. DeGrand and D. Toussaint (World Scientific, Singapore, 1989); "How to Renormalize the Schrödinger Equation", nnucl-th/9706029].

[49] H. W. Hammer and R. J. Furnstahl, Nucl. Phys. A 678 (2000) 277 arXiv:nucl-th/0004043.

[50] M. C. Rentmeester, R. G. Timmermans, J. L. Friar and J. J. de Swart, Phys. Rev. Lett. 82 (1999) 4992.

[51] A. D. Jackson, in Recent Progress in Many-Body Theories, vol. 3, ed. T. L. Ainsworth, C. E. Campbell, B. E. Clements, and E. Krotscheck (Plenum, 1992).

[52] A. D. Jackson and T. Wettig, Phys. Rep. 237 (1994) 325.

[53] H. Georgi and A. Manohar, Nucl. Phys. B234 (1984) 189.

[54] H. Georgi, Phys. Lett. B298 (1993) 187.

[55] J. L. Friar, Few Body Syst. 22 (1997) 161.

[56] J. L. Friar, D. G. Madland, and B. W. Lynn, Phys. Rev. C 53 (1996) 3085.

[57] C. J. Horowitz and B. D. Serot, Nucl. Phys. A 368, 503 (1981).

[58] H. Mueller and B. D. Serot, Nucl. Phys. A 606, 508 (1996)

[59] R. J. Furnstahl and H. W. Hammer, arXiv:nucl-th/0108069.

[60] C.-H. Lee, T. T. S. Kuo, G. Q. Li, and G. E. Brown, Phys. Rev. C 57 (1998) 3488.

[61] C. J. Horowitz, private communication.

[62] L. Engvik, M. Hjorth-Jensen, R. Machleidt, H. Muther, and A. Polls, Nucl. Phys. A627 (1997) 85.

[63] K. Sumiyoshi, K. Oyamatsu, and H. Toki, Nucl. Phys. A595 (1995) 327.

[64] Y. Hu, Ph.D. thesis, Indiana University, 2000.

[65] R. J. Furnstahl, H.-W. Hammer, and S. Puglia, in preparation.

[66] S. Karataglidis, K. Amos, B. A. Brown and P. K. Deb, arXiv:nucl-th/0111020. 
[67] B. C. Clark and S. Hama, in preparation.

[68] A. Trzcinska, J. Jastrzebski, P. Lubinski, F. J. Hartmann, R. Schmidt, T. von Egidy, and B. Klos, Phys. Rev. Lett. 87 (2001) 082501. 